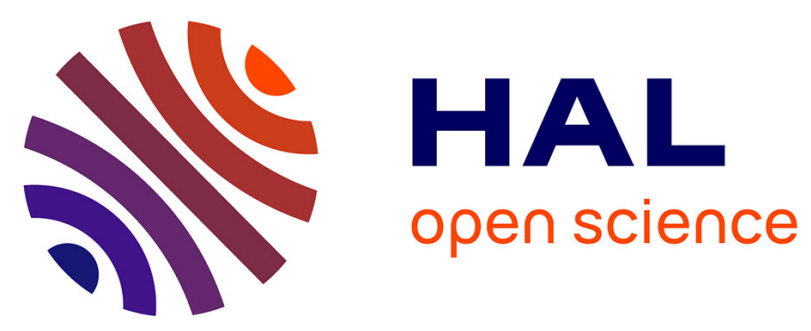

\title{
Le concept de champ magnétique et les phénomènes associés chez des étudiants du premier cycle à l'université
}

\author{
Roja Bagheri-Crosson, Patrice Venturini, Richard Lefevre
}

\section{To cite this version:}

Roja Bagheri-Crosson, Patrice Venturini, Richard Lefevre. Le concept de champ magnétique et les phénomènes associés chez des étudiants du premier cycle à l'université. Les Dossiers des sciences de l'éducation , 2002, 8, pp.23-32. halshs-00259988

\section{HAL Id: halshs-00259988 \\ https://shs.hal.science/halshs-00259988}

Submitted on 1 Mar 2008

HAL is a multi-disciplinary open access archive for the deposit and dissemination of scientific research documents, whether they are published or not. The documents may come from teaching and research institutions in France or abroad, or from public or private research centers.
L'archive ouverte pluridisciplinaire HAL, est destinée au dépôt et à la diffusion de documents scientifiques de niveau recherche, publiés ou non, émanant des établissements d'enseignement et de recherche français ou étrangers, des laboratoires publics ou privés. 
Bagheri, R., Venturini, P., \& Lefevre, R. (2002). Le concept de champ magnétique et les phénomènes associés chez des étudiants du premier cycle à l'université. Dossiers des Sciences de l'Education, 8, 23-32

Le concept de champ magnétique et les phénomènes associés chez des étudiants du premier cycle à l'université

BAGHERI Roja, LEMME, bât 3R1-b2, Université P. Sabatier, 118 Route de Narbonne, 31062 Toulouse cedex 04 tél. : 0561556810 ; fax 0561558319 ;

rbagheri@cict.fr

ATER.

VENTURINI Patrice, LEMME, bât 3R1-b2, Université P. Sabatier, 118 Route de Narbonne, 31062 Toulouse cedex 04 tél. : 0561556017 ; fax 0561558319 ;

patrice.venturini@cict.fr

Maître de conférences.

LEFÈVRE Richard, LEMME, bât 3R1-b2, Université P. Sabatier, 118 Route de Narbonne, 31062 Toulouse cedex 04 et IUFM du Pacifique, 15 rue de Verdun , BP MGA1, 98802 Nouméa cedex, Nouvelle Calédonie

tél. : 0561556810 ; fax 0561558319 ;

Lefevre@iufm-pacifique.nc

Professeur des universités, directeur de l'IUFM du Pacifique.

Résumé : L'étude proposée porte sur la manière dont est mobilisé le concept de champ magnétique, par des étudiants du DEUG Sciences de la Matière. Elle est décrite à partir d'un questionnaire soumis à 62 étudiants et d'entretiens qualitatifs menés avec six d'entre eux. Nous avons discerné des difficultés pour les étudiants de notre échantillon à prendre en compte la nature vectorielle du champ et à mettre en œuvre sa représentation ; notamment dans un espace à trois dimensions. Nous avons également remarqué que les propriétés du champ rassemblées dans les équations de Maxwell sont très peu explicitées, et ces équations ne semblent pas opératoires dans des situations simples.

Mots clés : Electromagnétisme, Maîtrise conceptuelle, Champ magnétique, Didactique des sciences.

Abstract : The proposed studies deal on the way first year students in « DEUG Sciences de la Matière » (first and second year of physics at university in France) mobilize the concept of electromagnetic field. It is described from a questionnaire submitted to 62 students and some qualitative interviews with six of them. We had discerned difficulties for the students of our sample to take into account the vector nature of the field and to build up a representation ; in particular in a three dimensional space. We had also notice that field properties collected in the Maxwell equations are not very often made explicit, and this equations don't seem operative in simple situations. 
Key words : Electromagnetism ; Conceptual mastery ; Magnetic field ; Didactics of science

\section{I) Introduction :}

L'article présente les premiers résultats d'un travail dont l'objectif est d'analyser la manière dont est mobilisé le concept de champ magnétique, par des étudiants du $1^{\mathrm{er}}$ cycle en Sciences de la Matière. Les travaux qui, en didactique de la physique, ont porté sur l'électromagnétisme sont rares. Venturini, Albe et Lascours (2000) ont montré que les étudiants ont des difficultés à donner un sens physique aux concepts fondamentaux de l'électromagnétisme et à établir des liens entre eux. Selon eux, les étudiants disposent de connaissances parcellaires, liées à une situation particulière. Maaroof et Benyamna (1997) trouvent que les élèves abordent l'apprentissage des phénomènes magnétiques avec des représentations construites avant et /ou pendant l'enseignement des sciences qui diffèrent de celles véhiculées par les modèles scientifiques. Greca et Moreira (1997) constatent que les étudiants utilisent en électromagnétisme des définitions et des formules qu'ils manipulent de manière routinière en vue de résoudre les problèmes, en témoignant d'une organisation cognitive très pauvre. Viennot et Rainson (1992) montrent que les étudiants, placés dans des situations où l'influence de plusieurs facteurs doit être prise en compte, privilégient un "raisonnement causal linéaire". Un des obstacles qu'elles ont repéré est la formule "champ si mobilité" qui traduit le fait que les étudiants raisonnent souvent comme si une cause n'existait qu'en cas d'effet manifeste. Cette manière de penser les conduit à refuser l'existence d'un champ en un point où les charges ne peuvent pas circuler. Aucun de ces travaux (à l'exception de Venturini, Albe et Lascours, 2000) n’ont porté sur la manière dont les étudiants appréhendent l'utilisation du concept de champ magnétique.

\section{II) Méthodologie :}

Pour cibler notre travail, nous avons décidé de commencer notre recherche par ce travail prospectif. Il vise à mettre en évidence des tendances générales dans l'utilisation de quelques concepts de l'électromagnétisme par les étudiants de DEUG et ce, à travers un premier questionnaire.

Tout d'abord, nous avons procédé à une détermination des éléments clés du domaine, dont la compréhension est indispensable aux étudiants pour appréhender tous les phénomènes électromagnétiques. Pour cela, nous avons examiné plusieurs ouvrages de physique destinés à l'enseignement universitaire (Perez J. PH., Carles R. et Fleckinger R., 1991, Purcell E.M., 1963, Feynman, 1979, Gié , Sarmant, 1985 et Benson, 1991) afin de repérer les points sur lesquels les auteurs insistent, ceux qui permettent des articulations entre différentes parties de l'électromagnétisme.

Un des auteurs (R. Fleckinger), professeur à l’Université Paul Sabatier, a validé les choix effectués au cours d'un entretien. Nous avons ensuite construit un questionnaire exploratoire comportant des situations et des questions (ouvertes et fermées) mettant en jeu ces éléments. 62 étudiants (en $4^{\text {ème }}$ semestre du DEUG Sciences de la Matière, mention physique) ont répondu à ce questionnaire pendant environ trente minutes à la fin de leur cours. Son analyse nous a permis, en fonction des réponses données, de choisir six étudiants avec lesquels nous avons procédé à des entretiens qualitatifs destinés à préciser certaines de leurs affirmations. Ces entretiens semi-directifs essentiellement exploratoires ont duré approximativement 45 minutes chacun.

\section{III) Résultats et interprétation :}


Comme nous l'avons déjà expliqué ci-dessus, les questions et situations proposées aux étudiants ont été choisies en fonction de ce que nous avons appelé : "éléments clés". Nous exposons ici uniquement les résultats concernant les questions relatives au champ magnétique. Nous estimons que les étudiants appréhendent le champ magnétique s'ils sont capables de définir sa nature (champ vectoriel), de définir ses propriétés de manière formelle (équation de Maxwell) et s’ils savent les utiliser dans quelques situations mettant en jeu les phénomènes électromagnétiques. Pour une meilleure compréhension, nous avons choisi de fournir ces résultats en pourcentage même si notre échantillon n'atteint pas cent étudiants.

\section{III.1) Nature du champ magnétique : Champ vectoriel :}

À la question "qu'est-ce qu'un champ vectoriel", 21\% des étudiants fournissent des réponses incomplètes ou proches de ce que l'on attend. Parmi ces étudiants, certains mentionnent une ou deux des caractéristiques des vecteurs et d'autres évoquent un espace où est appliqué un vecteur. 60\% des étudiants donnent des réponses erronées et enfin 19\% ne fournissent aucune réponse.

Nous remarquons tout d'abord que la notion de champ vectoriel est mal cernée par les étudiants puisqu'aucun ne fournit les éléments de la réponse que nous attendions d'eux c'est-àdire : "le champ est une grandeur physique, fonction des coordonnées de position d'un point de l'espace et du temps, que l'on peut représenter par un vecteur, utilisée pour décrire localement les propriétés de la matière ou pour interpréter les phénomènes qui s'y produisent." Tout au plus, certains font la liaison avec certaines des caractéristiques d'un vecteur, sans jamais mentionner les spécificités caractéristiques de la grandeur physique que l'on représente ainsi. C'est ce qu'ont d'ailleurs aussi constaté Albe et Venturini (2001). Il faut dire que le concept même de vecteur est beaucoup plus complexe qu'il n'y paraît. Selon une enquête (Fabre, 1992), 55\% des élèves de terminales ne connaissent pas l'ensemble des caractéristiques du vecteur. Lounis (1990) a montré que les élèves de Seconde privilégient l'aspect scalaire des grandeurs physiques vectorielles (en l'occurrence la force et la vitesse), par rapport à leur caractéristiques d'orientation spatiale. Ceci nous amène à nous interroger sur la façon dont l'étudiant " perçoit » le vecteur, sur le sens et la signification qu'ils donne à ce mot, pour la continuité de nos travaux.

Par ailleurs, si les étudiants sont capables d'associer à un point de l'espace une grandeur physique vectorielle, même s'ils n'y attribuent pas de signification, certains étudiants semblent avoir des difficultés à l'étendre à tous les points de l'espace et à comprendre que cette grandeur puisse prendre des valeurs différentes en différents points de l'espace.

Ainsi, par exemple, dans les réponses au questionnaire, on trouve à propos du champ vectoriel: "c'est une région où la valeur des vecteurs est la même". Au cours de l'entretien, un étudiant affirme "ne pas pouvoir imaginer des vecteurs en tous les points de l'espace", tandis qu'un autre réduit dans son propos le champ vectoriel à deux dimensions (dans un plan ).

D'autres étudiants définissent le champ vectoriel comme un "endroit" de l'espace, sans donner plus de précisions. Nous supposons qu'il existe donc pour certains d'entre eux, une confusion entre le champ lui même et la région de l'espace où on le considère.

Enfin plus de la moitié des réponses erronées sont du type : "C'est une zone de l'espace où est appliqué un vecteur. " Il semble y avoir une confusion entre le champ et sa représentation vectorielle.

Nous remarquons donc, pour ce qui concerne notre échantillon, que : 
1) Aucun étudiant ne donne une définition correcte du champ vectoriel ; cette notion est donc mal cernée.

2) Il y a une difficulté à concevoir le fait qu'en tous les points de l'espace, le champ puisse être différent.

3) Il semble y avoir une association, sinon une confusion, entre le champ lui même et l'endroit où on le considère.

4) Il semble également y avoir une confusion entre la grandeur physique et sa représentation vectorielle.

5) Il ne faut pas oublier le fait que la notion même de vecteur présente des difficultés.

\section{Champ magnétique :}

À la question: "dans une région de l'espace règne un champ magnétique (non variable dans le temps). Qu'est-ce que cela signifie pour vous?", 48\% des étudiants fournissent des réponses proches de ce que l'on attend. Parmi eux, certains évoquent une zone dans laquelle une particule chargée en mouvement est déviée et d'autres relient l'existence d'un champ magnétique à une force magnétique. Cette association entre champ et force a été également constatée lors des entretiens. D’autres évoquent la présence de sources de champ magnétique dans cette zone (aimants, courants) sans en décrire réellement les effets. 10\% des étudiants ne fournissent aucune réponse. Enfin, 42\% des réponses obtenues montrent des confusions ou bien sont tout simplement des reformulations de la question. Aucun étudiant ne qualifie le champ magnétique de champ vectoriel, ni n'évoque la déviation de l'aiguille d'une boussole sous l'effet d'un champ magnétique.

Nous considérons les réponses des étudiants comme correctes si, après avoir mentionné la nature vectorielle du champ magnétique, elles font référence au moins à un des éléments cidessous :

" Cette région de l'espace est située au voisinage d'un aimant ou d'un fil parcouru par un courant (champ défini à partir de sa source). Dans cette région de l'espace, un aimant est attiré ou repoussé, une boussole change d'orientation, des particules chargées en mouvement sont déviées de leur trajectoire d'origine (champ défini à partir de ses effets)."

La définition du champ par ses effets, c'est-à-dire par l'association faite entre champ magnétique et force ou couple de forces est pertinente lorsqu'un élément matériel ou un objet est susceptible d'interagir avec le champ. Il sera intéressant d'examiner si les étudiants considèrent que le champ existe en l'absence d'interaction. Nous rappelons qu'un des obstacles repérés pas S. Rainson (1995), est la formule "champ si mobilité"; en effet pour beaucoup d'étudiants, la cause n'existe qu'en cas d'effet manifeste. Cette dernière remarque, associée à l'absence de réponse à la question concernant le champ vectoriel nous laisse penser que la perception du champ magnétique reste floue, incertaine : les effets qu'il produit sont juste mentionnés et ne sont jamais décrits avec précision, pas plus que n'est mentionnée l'existence de ces effets en tous points de l'espace et ceci pour tous les étudiants interrogés.

\section{III.2) Propriétés du champ magnétique (Equations de Maxwell) :}

Les équations de Maxwell résument l'ensemble des phénomènes électromagnétiques d'où l'importance de leur compréhension.

En proposant ces équations sous forme intégrale et locale, nous avons demandé aux étudiants d'en fournir en quelques phrases une interprétation physique.

\section{Equation 1:}


$60 \%$ des étudiants interprètent l'équation $\operatorname{div} \vec{B}=0$ de manière incomplète comme traduisant la conservation du flux du champ magnétique. Seulement 23\% fournissent une interprétation correcte. En effet, ces derniers précisent que la conservation du flux du champ magnétique a lieu à travers une surface fermée. $17 \%$ des étudiants ne fournissent aucune réponse. Nous n'avons obtenu aucune interprétation en terme de lignes de champs fermées ou faisant allusion à l'absence de monopole magnétique. Les réponses fournies sont communes et apparaissent faire référence à une formulation classique et récurrente ; il semble qu'elle soit peu porteuse de sens. En liaison avec l'interprétation de cette équation, les étudiants avaient à identifier dans une autre question, le type de champ à partir de la représentation suivante en justifiant leur choix :



Champ électrostatique $\square$

Champ magnétostatique $\square$ Aucun des deux $\square$

Je ne sais pas

Les étudiants devaient identifier le champ magnétostatique en se référant aux éléments suivants : "cette représentation vectorielle présente une symétrie circulaire dans le plan considéré, les lignes de champ ne divergent pas à partir de leur source; elles semblent circulaires et paraissent se refermer." $37 \%$ des étudiants identifient un champ magnétostatique sans donner aucune justification ou justifient de manière erronée. Parmi eux, nous trouvons les deux tiers de ceux qui ont interprété la relation $\operatorname{div} \vec{B}=0$ de manière satisfaisante, mais ni les uns ni les autres n'ont pu justifier leur choix. L'équation $\operatorname{div} \vec{B}=0$, qui était rappelée dans le document, n'est pas opératoire dans cette situation concrète.

Par ailleurs, nous avons remarqué une « matérialisation » des lignes de champ magnétique. En effet, certains étudiants parlent des "lignes de champ en contact de l'aimant" lorsqu'ils avaient à identifier la représentation précédente du champ. Cette interprétation n'est qu'au stade d'hypothèse et nous prévoyons de la vérifier lors de nos prochains travaux. Nous rappelons qu'une étude (Törnkvist, 1992) concernant le champ électrique auprès de 566 étudiants de deuxième année à l'université a montré que les étudiants considèrent les lignes de champ comme une entité réelle et non comme une série de courbes représentant les propriétés vectorielles de l'espace. Visiblement, ces dernières sont peu connues des étudiants .

\section{Equation 2:}

En ce qui concerne l'équation $\operatorname{div} \vec{E}=\frac{\rho}{\varepsilon_{0}}$, nous attendions des étudiants qu'ils fournissent la réponse suivante : "le flux du champ électrique à travers une surface fermée est créé à partir de la somme des charges à l'intérieur de cette surface ; il dépend d'عo qui est caractéristique du milieu qui "accueille" le champ." Même si cette équation ne concerne pas directement le champ magnétique ou magnétostatique, son interprétation permet de différencier les 
propriétés de ce dernier avec celles du champ électrostatique. C'est pour cette raison que nous l'avons prise en considération.

49\% des étudiants ne font que mentionner le nom de l'équation (Gauss) ou que la formuler en langage naturel ; $29 \%$ ne fournissent aucune réponse et $19 \%$ fournissent des réponses erronées, certaines de ces réponses inversant la cause et l'effet. Seuls 3\% des étudiants évoquent la source du champ électrostatique. Aucun étudiant n'a interprété l'équation en terme de lignes de champ divergentes à partir d'une charge ou convergentes vers une charge. En liaison avec l'interprétation de cette équation, les étudiants avaient également à identifier dans une autre question, le type de champ à partir de la représentation suivante et justifier leurs choix :

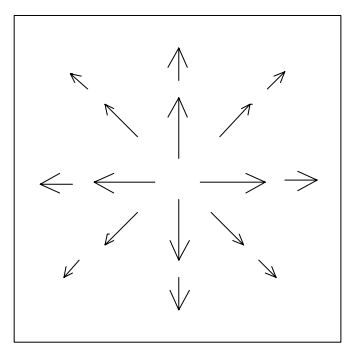

Champ électrostatique $\square$

Champ magnétostatique $\square$

Aucun des deux $\square$

Je ne sais pas

En remarquant la forme des lignes de champ, les étudiants devaient identifier le champ comme le champ électrostatique d'une charge positive située au centre de la figure, ce que $25 \%$ ont fait sans donner aucune justification ou en justifiant de manière erronée. Les 3\% qui ont évoqué la source du champ électrique concernant l'équation Maxwell- Gauss sont dans ce cas. Nous constatons ici aussi que l'équation de Maxwell n'est pas opérationnelle dans cette situation concrète.

Ainsi, nous pouvons supposer que les étudiants qui identifient le champ électrostatique ou magnétostatique le font peut-être à partir de souvenirs visuels ne référant pas aux propriétés des champs.

\section{Equation 3:}

$44 \%$ des réponses concernant l'équation $\operatorname{rot} \vec{B}=1 / c 2(\partial \vec{E} / \partial t)+\mu_{0} j$ indiquent qu'il y a une "dépendance" ou "proportionnalité" entre $\vec{E}$ et $\vec{B}$ sans apporter plus de précision, ce qui constitue une lecture de l'équation au premier niveau. 23\% citent uniquement le nom de l'équation (Maxwell- Ampère). 29\% ne fournissent aucune réponse. Seulement 4\% des réponses, relatives à la source du champ magnétique sont satisfaisantes : "la variation $d u$ champ électrique produit un champ magnétique."

\section{Equation 4 :}

Quant à l'équation $\operatorname{rot} \vec{E}=-\partial \vec{B} / \partial t, 44 \%$ ne répondent pas. $24 \%$ proposent une simple reformulation de l'équation. 3\% des étudiants rappellent uniquement le nom de l'équation (Faraday). $7 \%$ des réponses obtenues évoquent de manière exacte le phénomène d'induction sans aucune autre explication. Seulement $22 \%$ des étudiants fournissent une interprétation correcte de l'équation en rappelant le couplage qui existe entre $\vec{E}$ et $\vec{B}$.

Par ailleurs, nous avons remarqué une «matérialisation » du champ magnétique. En effet, certains d'entre eux traduisent $\partial \vec{B} / \partial t$ en "déplacement du champ" ou "champ en mouvement" . 
Nous avons aussi testé la compréhension de l'équation de Maxwell-Faraday dans deux situations qui imposaient son utilisation pertinente pour les interpréter :

"En 1820, Ersted découvre qu'un courant électrique continu produit un champ magnétique (permanent). De nombreux chercheurs (avant Faraday et Henry) ont essayé de montrer l'existence de l'effet inverse mais sans succès. Pourquoi?"

Seulement $16 \%$ des étudiants ont donné une réponse correcte à cette question en affirmant que si $\vec{B}$ ne varie pas dans le temps, il ne peut y avoir de champ électrique et donc de courant.

" Une particule chargée est initialement immobile dans un champ magnétique vertical, uniforme et variable dans le temps (on néglige le champ gravitationnel). Que se passe-t-il?" Nous attendions des étudiants la réponse suivante : " La particule se met en mouvement. En effet, le champ magnétique variable crée un champ électrique qui déplace la charge. Le mouvement de la charge dépend donc de la configuration du champ électrique induit, donc du champ magnétique variable."

Seulement une personne a fourni une bonne réponse avec une justification correcte.

Nous constatons donc que le sens physique des opérateurs mathématiques et le sens des équations sont peu ou pas connus des étudiants. Venturini, Albe et Lascours, (2000) avaient, dans un autre contexte, fait une constatation du même type en montrant que les étudiants utilisent les outils mathématiques de manière procédurale sans leur donner un sens physique. Enfin, nous constatons que les équations de Maxwell, qu'elles soient interprétées ou non, sont peu opératoires sur les situations concrètes que nous avons proposées.

\section{IV) Conclusions :}

L'enquête sur ces 62 étudiants nous a permis de constater qu'ils éprouvent de réelles difficultés à appréhender le champ magnétique et ses propriétés. Les premières difficultés résident dans la perception de propriétés physiques de points immatériels de l'espace et dans la compréhension de la nature de ces propriétés rassemblées dans la notion de champ (vectoriel).

Il est difficile pour les étudiants de se représenter un champ, une grandeur non matérialisée sur un espace à trois dimensions. Si certains d'entre eux arrivent à avoir une représentation à une ou deux dimensions, ils éprouvent des difficultés dès lors qu'il s'agit de l'espace à trois dimensions. Nous supposons également que certains d'entre eux utilisent un support "substantialiste" pour se représenter le champ magnétique. Nous pensons qu'il s'agit ici d'une représentation substantialiste plutôt qu'un obstacle substantialiste au sens de Bachelard (1980). Nous envisageons de vérifier cette supposition lors de notre prochaine enquête.

Nous avons constaté que les étudiants de notre échantillon éprouvent beaucoup de difficulté à utiliser les outils mathématiques. Ils semblent ignorer le sens physique de ces opérateurs. Quant aux équations de Maxwell, la majorité d'entre eux n'en a retenu que le nom.

La compréhension du concept de champ est indispensable pour appréhender tous les phénomènes électromagnétiques ; c'est la raison pour laquelle la suite de notre travail sera centrée sur l'étude de la manière dont est mobilisé ce concept par les étudiants, alors qu'ici nous avons examiné que leurs connaissances quasi- formelles sur la nature et les propriétés du champ magnétique, premier moyen pour nous d'aborder l'usage de ce concept. En se référant aux travaux sur les concepts et leurs constructions (Vergnaud G., 1990, Lemeignan G. et Weil-Barais A., 1993), nous examinerons dans des situations différentes quant à leur nature (formelle, expérimentale...) et à leur objet (représenter, prévoir, interpréter...) les 
représentations mentales des étudiants et les liens qu'elles entretiennent avec les représentations symboliques.

Nous examinerons aussi d'un point de vue épistémologique en réponse à quels besoins ce concept a été introduit et de quelle manière cela a été fait. En dégageant de l'histoire de l'électromagnétisme les débats scientifiques (Oersted, Ampère, Faraday, Maxwell...), les controverses et les changements de paradigmes (au sens de T. Kuhn) suscités par la construction du concept de champ magnétique et ses concepts associés, nous pouvons comprendre les difficultés conceptuelles qui ont retardé l'élaboration du savoir concerné. En effet, de nombreux travaux en didactique (ex : Seroglou, Koumaras et Tselfes, 1998, Giannetto, 1993) ont montré que l'histoire des sciences peut être une source fructueuse d'information susceptible d'éclairer les difficultés de l'apprenant dans la formation et l'utilisation des concepts scientifiques.

\section{V) Bibliographie :}

Albe V. \& Venturini P. (2001). Concepts électromagnétiques : absence de sens et manque de structuration chez les étudiants. In SCHOLE, numéro hors-série 2001, Actes des 2ièmes rencontres scientifiques de l'ARDIST, "Actualité de la recherche en didactique des sciences expérimentales et des techniques", Carry le Rouet, 17-20 octobre 2001, pp.241-252. Marseille : IUFM.

Bachelard G. (1980). La formation de l'esprit scientifique, Paris : Vrin.

Bar V., Zinn B. \& Rubin E. (1997). Children's ideas about action at distance. International Journal Science of Education, 19, (10), 1137-1157.

Benson H. [1991]. Physique 2, Electricité et Magnétisme, De Boeck Université (1999).

Fabre P. (1992). Image mentale du vecteur. Mémoire de DEA, LEMME, Université Paul Sabatier, Toulouse.

Feynman R. (1979). Cours de physique de Feynman, électromagnétisme, Paris : InterEdition.

Galili I. (1995). Mechanics background influences studients's conceptions in electromagnetism. International Journal Science of Education, 17 (3), 371-387.

Giannetto E. (1993). The Impetus Theory : Between History of Physics Teaching and Science Education. Science \& Education, 2, 239- 242.

Gié H., Sarmant J.P. (1985). Electromagnétisme . Paris : Technique et Documentation.

Greca M. \& Moreira M. (1997). The kinds of mental representation - models propositions and images - used by college physics students regarding the concept of field, 19 (6), 711-724.

Lemeignan G., Weil-Barais A. (1993). Construction des concepts en physique, Paris : Hachette éducation.

Lounis A. (1990). L'introduction aux modèles vectoriels en physique et en mathématique : conception et difficultés des élèves, essais de remédiation. Thèse, Université d'Aix Marseille I. 
Maarouf A., Benyamna S. (1997). La construction des sciences physiques par les représentations et les erreurs : cas des phénomènes magnétiques. Didaskalia, 11, 101-118.

Perez J. PH., Carles R. et Fleckinger R. (1991). Electromagnétisme, vide et milieux matériels, Paris : Masson.

Purcell E.M. [1963]. Cours de physique Berkeley, électricité et magnétisme, Dunod (1998).

Törnkvist S., Petersson K.A. et Tranströmer G. (1993). Confusion by representation : On comprehension of electric field concept. American association of Physics Teachers, 61 (4), 335-338.

Rainson S. (1995) Superposition des champs électrique et causalité. Thèse, Université Paris 7.

Seroglou F., Koumaras P. et Tselfes V. (1998). History of Science and Instructional Design : The case of Electromagnetism. Science \& Education, 7, 261- 280.

Venturini P., Albe V. \& Lascours, J. (2000). Rapport des étudiants au champ et au flux magnétiques. In A. Chabchoub (Eds). Actes du $5{ }^{\text {ème }}$ Colloque International de didactique et d'épistémologie des sciences Rapports au savoir et apprentissage des sciences , 7-8-9 avril 2000 Sfax (pp.175-186). Tunis : ATRD.

Vergnaud G. (1990) La théorie des champs conceptuels. Recherche en Didactique des Mathématiques, 10 (23), 133- 170.

Viennot L. Rainson (1992) Students' reasoning about the superposition of electric field. International Journal Science of Education, 14 ( 4 ), 475-487. 